University of Nebraska - Lincoln

DigitalCommons@University of Nebraska - Lincoln

2014

Temporary Anion States of Three Herbicide Families

Adam M. Scheer

Kayvan Aflatooni

Gordon A. Gallup

Paul Burrow

Follow this and additional works at: https://digitalcommons.unl.edu/physicsburrow

Part of the Atomic, Molecular and Optical Physics Commons

This Article is brought to you for free and open access by the Research Papers in Physics and Astronomy at DigitalCommons@University of Nebraska - Lincoln. It has been accepted for inclusion in Paul Burrow Publications by an authorized administrator of DigitalCommons@University of Nebraska - Lincoln. 


\title{
Temporary Anion States of Three Herbicide Families
}

\author{
A. M. Scheer, K. Aflatooni, G. A. Gallup, and P. D. Burrow
}

Department of Physics and Astronomy, University of Nebraska-Lincoln, Lincoln, Nebraska 68588-0299, United States

Corresponding author - P. D. Burrow: email pburrow@unl.edu

Present Addresses - A. M. Scheer, Combustion Research Facility, Sandia National Laboratories, Livermore, CA 94551-0969, USA; K. Aflatooni, Department of Physics, Fort Hays State University,

Hays, KS 67601-4099, USA.

\begin{abstract}
Electron scattering studies are used to locate the energies of temporary negative ion states of three chloro-substituted molecular families of herbicidal importance: salicylic and phenoxyacetic acids and acetamides. The correlation between these energies and the computed virtual orbital energies of the compounds is examined and used to put the latter on an absolute energy scale. Such scaling of orbital energies permits the anion states of other members of these families, for which experimental data may not be available, to be estimated from the calculated orbital energies. Studies of electron reduction rates often rely on calculated LUMO energies as molecular descriptors. The use of measured anion energies as well as appropriately scaled orbital energies should serve to improve such studies in these and in related herbicides.
\end{abstract}

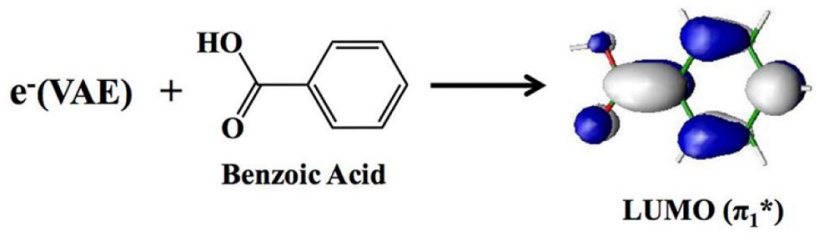

Published in The Journal of Physical Chemistry A 118 (2014), pp 7242-7248.

Special Issue: Kenneth D. Jordan Festschrift

doi:10.1021/jp409345m

Copyright (C) 2013 American Chemical Society. Used by permission.

Submitted September 18, 2013; revised November 12, 2013; published November 21, 2013 


\section{Introduction}

Electron transfer to chlorine-bearing pollutants such as substituted alkanes and alkenes is known to assist in dehalogenating such species. Electron occupation of a low-lying normally empty $\sigma^{*}(\mathrm{C}-\mathrm{Cl})$ orbital in these compounds leads to efficient ejection of chloride by virtue of the antibonding character of the orbital and the large electron affinity of the chlorine atom. A brief discussion of the important characteristics of temporary anion states in hydrocarbons may be found elsewhere. ${ }^{1}$ In unsaturated nonplanar chlorinated hydrocarbons, the mechanism may be further complicated by low-lying orbitals of $\pi^{*}(\mathrm{C}=\mathrm{C})$ local symmetry that can mix with the $\sigma^{*}(\mathrm{C}-\mathrm{Cl})$ orbital. ${ }^{2}$ Even in planar compounds, such $\pi^{*} / \sigma^{*}$ mixing may take place by out-of-plane vibrational distortions. ${ }^{3}$ The energies and symmetries of the lowest few anion states, some or all of which may be temporary anion states, are therefore of significance in remediation studies.

A key parameter in dissociative electron attachment (DEA) processes is the energy required to inject an electron into one of the normally empty orbitals of the molecular species in its neutral equilibrium geometry, the vertical attachment energy (VAE). In studies relating reductive rate constants to molecular properties, such as quantitative structure-activity relationships (QSARs), computed virtual orbital energies (VOEs) for the lowest unoccupied molecular orbitals (LUMOs) are often used as a measure or "descriptor" of the electron accepting property of the molecular species. VOEs are relatively easy to calculate with currently available quantum chemical programs using either Hartree-Fock (HF) or density functional theory (DFT) approaches. Such VOEs are functions of the computational method and have no physical meaning, although their relative values can be of use. Correlations between experimentally determined VAEs and calculated VOEs of $\pi^{*}$ orbitals, however, are not the same for different molecular families. ${ }^{4-6}$ Furthermore, even within a series of structurally similar molecules, it is necessary to treat orbitals of different character separately. For example, the $\pi^{*}(\mathrm{C}=\mathrm{C})$ VOEs of chlorinated alkenes correlate with measured VAEs in a different manner than do the $\sigma^{*}(\mathrm{C}-\mathrm{Cl})$ VOEs. Different substituents such as chlorine and bromine are also observed to correlate differently with VAEs, ${ }^{7,8}$ as do $\sigma^{*}$ anion states of different families of compounds, such as group 14 dimers. ${ }^{9}$ However, 
it has been shown in a number of studies that by shifting and scaling VOEs to match measured VAEs in a given family of molecules, a "correction" equation may be obtained and applied to other members of this family whose VAEs are not available. Such scaled VOEs (SVOEs) possess greater internal consistency and may be used to predict unknown VAEs. A recent paper ${ }^{10}$ illustrates examples in which the correlations between reduction rates and LUMO energies are altered by use of measured VAEs or by SVOEs determined from the scaling equations based on VAEs in related compounds. The appendix of that paper contains a listing of scaling equations known to us that have been employed.

Although LUMO energies have received the major focus in QSAR studies, it is worth stressing that in comparisons among several molecular families, for example, chlorinated alkenes and alkanes, the LUMOs may have different symmetries ${ }^{10,11}$ and thus it may be important to have VAE or SVOE data for several of the lowest anion states in a given compound. Furthermore, because it is likely that VAE data may not be available for a particular compound, it is important to have VAE data in as many significant molecular families as possible to employ as "training compounds" for the construction of SVOEs in the related compounds of interest. For these reasons, we describe here our electron transmission spectroscopy (ETS) ${ }^{12}$ studies of three families of chlorinated compounds whose properties are of environmental interest as herbicides, ${ }^{13}$ namely, salicylic and phenoxyacetic acids, and acetamides.

Following a brief discussion of the experimental technique, we present our results in 3-, 4-, and 5-chlorosalicylic acids along with the prototypes benzoic and salicylic acid. In addition to the VAEs, we discuss the presence of an additional feature in the transmission spectra, a vibrational Feshbach resonance (VFR), observed in the compounds that have supercritical electric dipole moments. In the subsequent sections, we report results in phenoxyacetic acid and its $p$-chloroand $o$-chlorosubstituted forms and the methyl esters of (2,4-dichlorophenoxy)acetic acid (2,4-D) and (2,4,5- trichlorophenoxy)acetic acid. Lastly, we consider acetamide, 2-chloroacetamide, acetanilide, propachlor, and metolachlor. 


\section{Experimental methods}

In ETS, an energy-selected and magnetically collimated electron beam is transmitted through a collision cell containing the sample gas. Scattered electrons are rejected at a retarding electrode following the collision cell, and the unscattered electrons are collected. To accentuate resonant structure in the total cross section, the electron energy within the cell is modulated with a small sinusoidal voltage and the ac component of the transmitted current is sent to a lock-in amplifier, enabling detection of the derivative of transmitted current with respect to energy. ${ }^{12}$ In the derivative signal, a peak in the total cross section is signaled by a minimum followed by a maximum. The resonance energy is assigned to the vertical midpoint of the dip to peak structure. Because the attachment process is rapid relative to nuclear motion, the resonance energy characterizes the molecule in its neutral equilibrium geometry and is a measure of the VAE.

A peak at $2.46 \mathrm{eV}$ in the derivative signal of $\mathrm{N}_{2}$ associated with the $v$ $=2$ vibrational level of the ${ }^{2} \Pi_{\mathrm{g}}$ anion state was used to calibrate the energy scale in this work. The electron beam had a typical energy width of $40 \mathrm{meV}$ (fwhm) and was generated by a trochoidal electron monochromator. ${ }^{14}$ The uncertainty in the energy of a narrow resonance is taken to be $\pm 50 \mathrm{meV}$. Samples with sufficient vapor pressure at room temperature were introduced into the collision cell via a leak valve external to the vacuum chamber. An oven directly attached to the collision chamber was employed for samples with low vapor pressures. Sample temperatures ranged from 88 to $100{ }^{\circ} \mathrm{C}$ to achieve suitable electron beam attenuation. The collision cell temperatures were maintained $50-100{ }^{\circ} \mathrm{C}$ higher to keep the electrodes clean.

\section{Chlorosalicylic acid family}

\section{i. Electron Transmission Spectra.}

Figure 1 displays the ET spectra of benzoic and salicylic acid, serving as prototypes of the substituted acids. The spectrometer is normally operated with the retarding voltage as close as possible to the voltage of the filament, subject to instrumental effects discussed elsewhere, ${ }^{15}$ to achieve measurements reflecting the shape of the total 


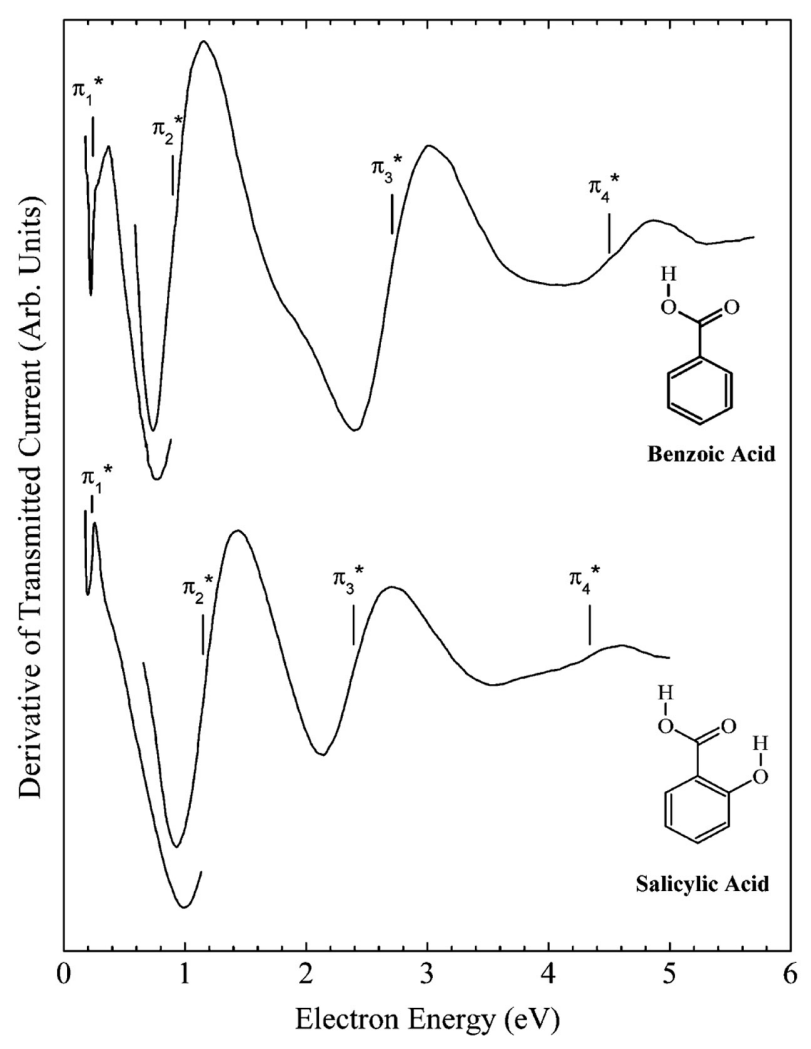

Figure 1. Derivative with respect to energy of the electron current transmitted through benzoic and salicylic acid as a function of electron energy. The vertical lines show the energies assigned to the occupation of the indicated orbitals.

scattering cross section. Different settings, resulting in the two overlapping curves, were required at very low energy and at higher energy to avoid artifacts in the spectra. The energies of the midpoints of the four resonances observed in each compound, that is, their VAEs, are shown as short vertical lines and listed in Table 1. Similar to the case for the spectrum of styrene, ${ }^{16}$ substitution of an unsaturated group, $\mathrm{COOH}$ in this case, on a benzene ring to produce benzoic acid breaks the degeneracy of the benzene $\mathrm{e}_{2 \mathrm{u}}$ orbital, yielding a low lying resonance, $\pi_{1}^{*}$, made up of the bonding combination of the $b_{1}$ ring $\pi^{*}$ and the $-\mathrm{COOH} \pi^{*}$ components. The antibonding combination, $\pi_{3}{ }^{*}$, is pushed to higher energy. The $\pi_{2}{ }^{*}$ resonance lies on the ring, whereas $\pi_{4}{ }^{*}$ is distributed over the entire molecule in an antibonding fashion. Figure 2 shows the four $\pi^{*}$ molecular orbitals corresponding to these resonances. Salicylic acid possesses an ET spectrum similar to that of benzoic acid and analogous assignments are given. 
Table 1. Empty Orbital Designations, Calculated VOEs, and Measured VAEs of the Salicylic Acid Family

\begin{tabular}{|c|c|c|c|}
\hline compound & orbital & $\begin{array}{c}V O E \\
(\mathrm{eV})\end{array}$ & $\begin{array}{l}V A E \\
(\mathrm{eV})\end{array}$ \\
\hline \multirow[t]{5}{*}{ benzoic acid } & $\pi_{1}^{*}[\phi+\mathrm{HCOOH}]^{a}$ & 2.6150 & 0.26 \\
\hline & $\pi_{2}^{*}\left[\phi\left(“ \mathrm{a}_{2} ”\right)\right]$ & 3.7497 & 0.90 \\
\hline & $\sigma_{1}^{*}(\mathrm{OH})$ & 6.1770 & N.O. \\
\hline & $\pi_{3}{ }^{*}[\phi-\mathrm{HCOOH}]^{a}$ & 6.7539 & 2.71 \\
\hline & $\pi_{4}^{*}[\phi]$ & 10.4437 & $4 \cdot 5$ \\
\hline \multirow[t]{5}{*}{ salicylic acid } & $\pi_{1}^{*}$ & 2.4926 & 0.17 \\
\hline & $\pi_{2}^{*}$ & 4.1334 & 1.15 \\
\hline & $\sigma_{1}^{*}(\mathrm{OH})$ & 6.0545 & N.O \\
\hline & $\pi_{3}^{*}$ & 6.4382 & 2.39 \\
\hline & $\pi_{4}^{*}$ & 10.395 & $4 \cdot 4$ \\
\hline \multirow[t]{7}{*}{ 3-chlorosalicylic acid } & VFR & & 0.31 \\
\hline & $\pi_{1}^{*}$ & 2.1307 & N.O \\
\hline & $\pi_{2}^{*}$ & 3.7552 & 0.86 \\
\hline & $\sigma_{1}^{*}(\mathrm{C}-\mathrm{Cl})$ & 5.3632 & N.O \\
\hline & $\sigma_{2}^{*}(\mathrm{OH})$ & 6.0083 & N.O \\
\hline & $\pi_{3}^{*}$ & 6.1062 & 2.08 \\
\hline & $\pi_{4}^{*}$ & 9.9757 & N.O \\
\hline \multirow[t]{7}{*}{ 4-chlorosalicylic acid } & VFR & & 0.38 \\
\hline & $\pi_{1}^{*}$ & 2.1443 & N.O \\
\hline & $\pi_{2}^{*}$ & 3.7252 & 0.79 \\
\hline & $\sigma_{1}^{*}(\mathrm{C}-\mathrm{Cl})$ & 5.1892 & N.O. \\
\hline & $\sigma_{2}^{*}(\mathrm{OH})$ & 5.9620 & N.O \\
\hline & $\pi_{3}^{*}$ & 6.0600 & 2.10 \\
\hline & $\pi_{4}^{*}$ & 10.226 & 4.2 \\
\hline \multirow[t]{6}{*}{ 5-chlorosalicylic acid } & $\pi_{1}^{*}$ & 2.1116 & N.O \\
\hline & $\pi_{2}^{*}$ & 3.6871 & 0.77 \\
\hline & $\sigma_{1}^{*}(\mathrm{C}-\mathrm{Cl})$ & 5.2572 & N.O \\
\hline & $\sigma_{2}^{*}(\mathrm{OH})$ & 5.9457 & N.O \\
\hline & $\pi_{3}^{*}$ & 6.0899 & 2.06 \\
\hline & $\pi_{4}^{*}$ & 10.204 & N.O \\
\hline
\end{tabular}

a. The ring $\pi^{*}$ combined in a bonding $(+)$ or antibonding $(-)$ manner with the $-\mathrm{HCOOH} \pi^{*}$.

b. Not Observed. 


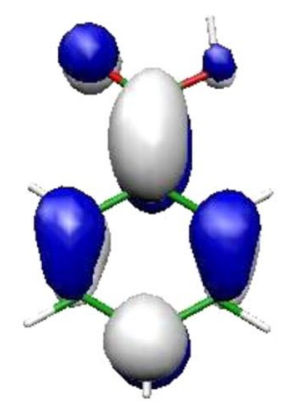

$\pi_{1}^{*}$ (LUMO)

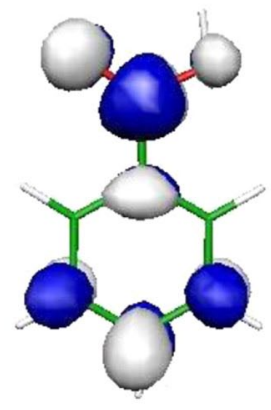

$\pi_{3}^{*}(\mathrm{LUMO}+4)$
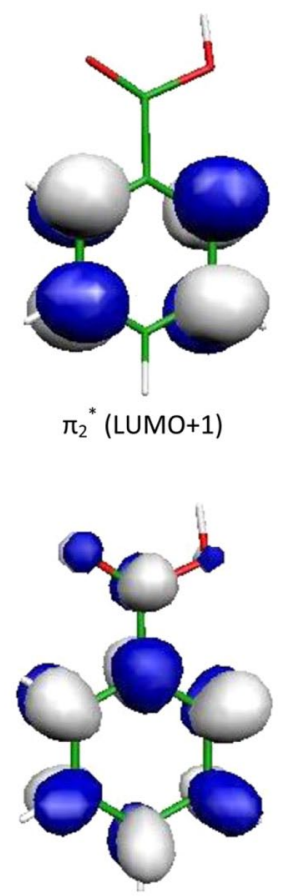

$\pi_{4}^{*}(\mathrm{LUMO}+9)$

Figure 2. Four lowest $\pi^{\star}$ molecular orbitals of benzoic acid.

Figure 3 shows the results in the $3^{-}, 4^{-}$, and 5-chloro-substituted salicylic acids, with the unsubstituted compound shown again for comparison. We have omitted the upper energy range because the $\pi_{4}{ }^{*}$ resonances were weak and difficult to locate in the 3- and 5-chloro compounds. As is apparent in Figure 3, addition of a chlorine atom stabilizes the $\pi^{*}$ anion states substantially, relative to those of salicylic acid. The anion state corresponding to occupation of the LUMO was not observed in the ET spectra of any of the three chlorosalicylic acids. These states, if resonances, may be obscured by the turn-on of the electron beam with its finite resolution and also from low-energy scattering by the molecular dipole moment. Alternatively, they may be stable anion states and thus inaccessible by ETS.

HF calculations using the $6-31 \mathrm{G}(\mathrm{d})$ basis set were employed to optimize the geometries, determine electric dipole moments, and compute VOEs of the compounds in this work. The calculations were carried out with GAMESS. ${ }^{17}$ Table 1 summarizes the observed VAEs and 


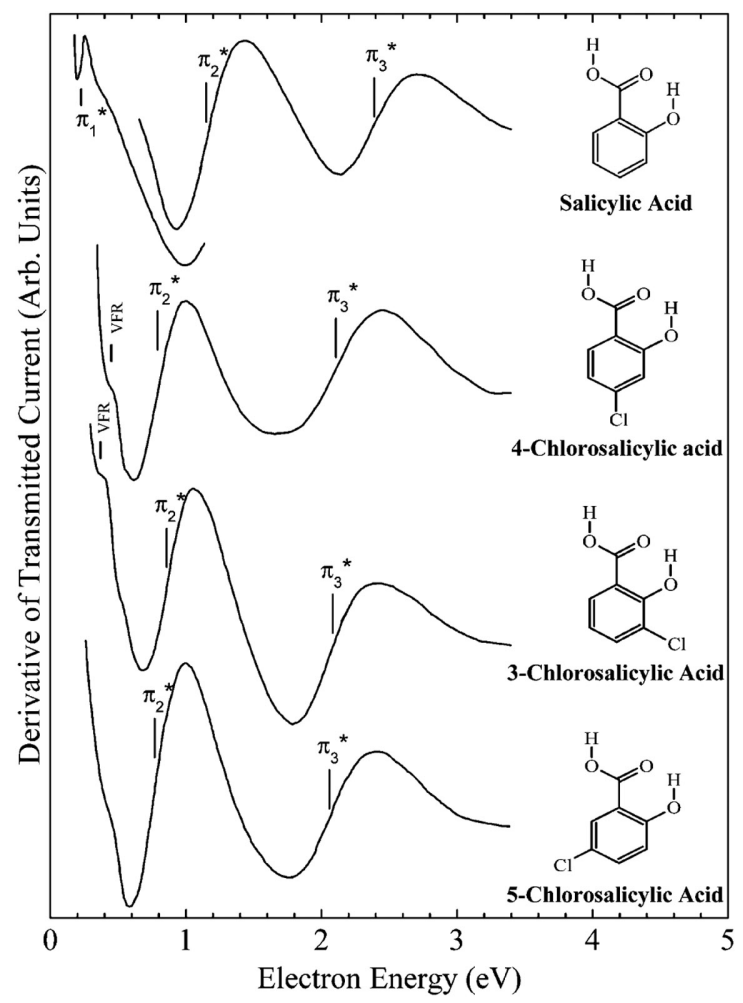

Figure 3. As in Figure 1 for salicylic acid and the three monochlorosalicylic acids.

calculated VOEs for the benzoic and salicylic acids. The features indicated by VFR will be discussed later. To illustrate the correlation between these quantities, in Figure 4 we have plotted the VAEs vs the VOEs of the five compounds in Table 1. A linear regression yields the following: $\mathrm{VAE}=(\mathrm{VOE}-2.12) / 1.87$, with all quantities in electronvolts. This regression could be used to shift and scale calculated VOEs of other members of the salicylic acid family to yield predicted VAEs (SVOEs). The use of such equations for VOE scaling is subject to several caveats, not always noted. First, according to the theoretical study of Chen and Gallup, 5 the VOE/VAE relationship is not strictly linear, although over narrow energy ranges a linear approximation is adequate. Second, the higher lying shape resonances, above $\sim 3 \mathrm{eV}$ in our compounds, may be shifted in energy by mixing with two-particle one-hole states, that is, with doubly excited states of the anion, of the same symmetry. Third, the role of different conformers may need to be considered in sufficiently flexible molecules. 


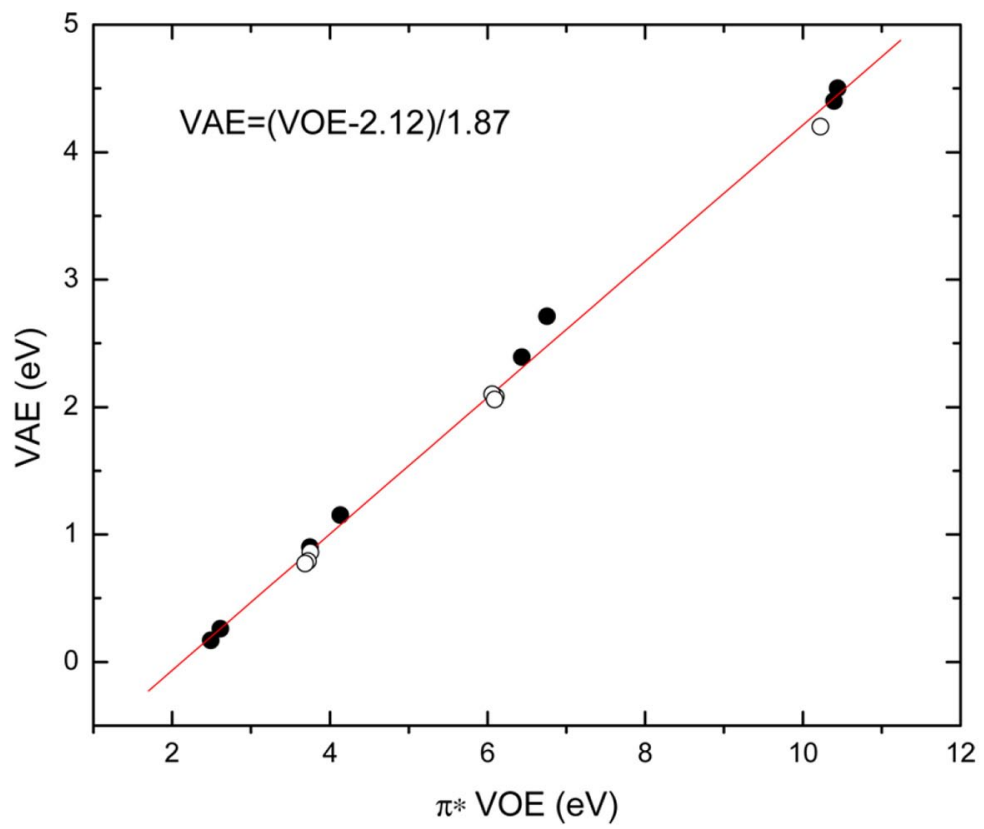

Figure 4. Measured VAEs as a function of HF/6-31G(d) calculated VOEs in the salicylic acid family (Table 1). The linear regression and its equation are shown. The closed circles indicate benzoic and salicylic acid. Open circles show the chlorosalicylic acids.

Using the regression given above, the predicted VAEs of the ground anionic states of the Cl-substituted salicylic acids fall within $\pm 13 \mathrm{meV}$ of $\mathrm{o} \mathrm{eV}$, and thus the regression is not sufficiently accurate to determine whether these states are stable or not. However, we note that the $\pi_{2}{ }^{*}$ and $\pi_{3}{ }^{*}$ VAEs of the chloro-substituted compounds are more stable relative to those of salicylic acid by $0.29-0.38 \mathrm{eV}$, with an average value of $0.33 \mathrm{eV}$. If this degree of stabilization occurs in the $\pi_{1}^{*}$ VAEs of the monochloro compounds, their VAEs could be approximately 0.17 $-0.33=-0.16 \mathrm{eV}$, implying that their ground state anions are stable.

Resonances owing to occupation of the $\sigma^{\star}(\mathrm{C}-\mathrm{Cl})$ orbital are readily seen in the chlorobenzenes, ${ }^{18}$ as well as in saturated halo-carbons. ${ }^{7}$ The $\pi^{*}$ anion scalings described here are inappropriate for such $\sigma^{*}(\mathrm{C}-\mathrm{Cl})$ anion states. However, we can approximate the locations of the latter using a scaling determined earlier for chloroalkanes in which these anion states are observed: ${ }^{7} \mathrm{SVOE}=(\mathrm{VOE}-2.83) / 1.11$. Applying this to the $\sigma^{*}(\mathrm{C}-\mathrm{Cl})$ VOEs given in Table 1 yields $2.28,2.13$, and $2.19 \mathrm{eV}$ for the 3-, 4-, and 5-chloro compounds, respectively. Unfortunately these broad resonances are centered at nearly the same energies as the $\pi_{3}{ }^{*}$ 
resonances and are completely obscured by them. They may, however, play a role in the dissociative electron attachment cross sections, contributing near the energies of the lowest $\pi^{*}$ resonances by virtue of $\pi^{*} / \sigma^{*}$ coupling owing to out-of-plane chlorine motion. ${ }^{3}$

\section{ii. Vibrational Feshbach Resonances.}

Figure 5 shows an expanded view of the low-energy portion of the ET spectrum in the three chloro-substituted compounds and lists their calculated dipole moments in Debye. We note that the moments of the $3-\mathrm{Cl}$ and $4-\mathrm{Cl}$ compounds are supercritical, above $\sim 2.5 \mathrm{D},{ }^{19}$ which is sufficient to bind an electron in a diffuse orbital of $\sigma$ symmetry, leading to a dipole bound state (DBS). The positive end of the moment is on the side closest to the $\mathrm{OH}$ moiety on the $-\mathrm{COOH}$ group, and we expect the greatest electron density in this region. Such a DBS will mix with the low-lying temporary valence anion states of $\Sigma$ symmetry and the coupling will be greatest when the wave functions of the latter

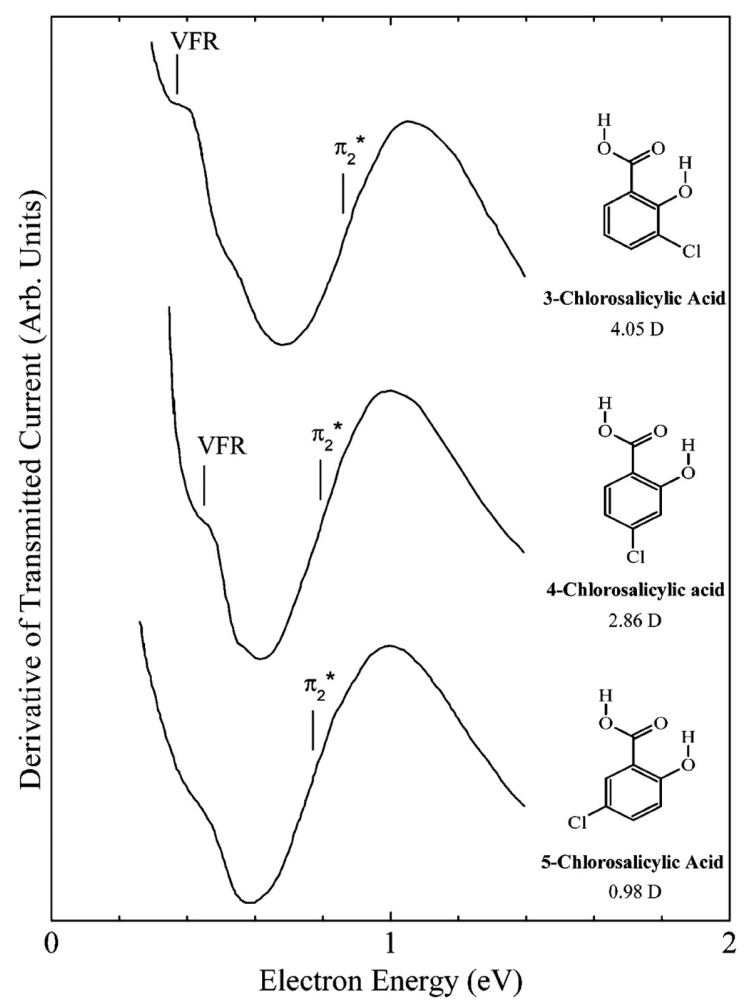

Figure 5. Expanded view of the transmission spectra below $1 \mathrm{eV}$ showing the structure assigned to vibrational Feshbach resonances in the two compounds with supercritical dipole moments. 


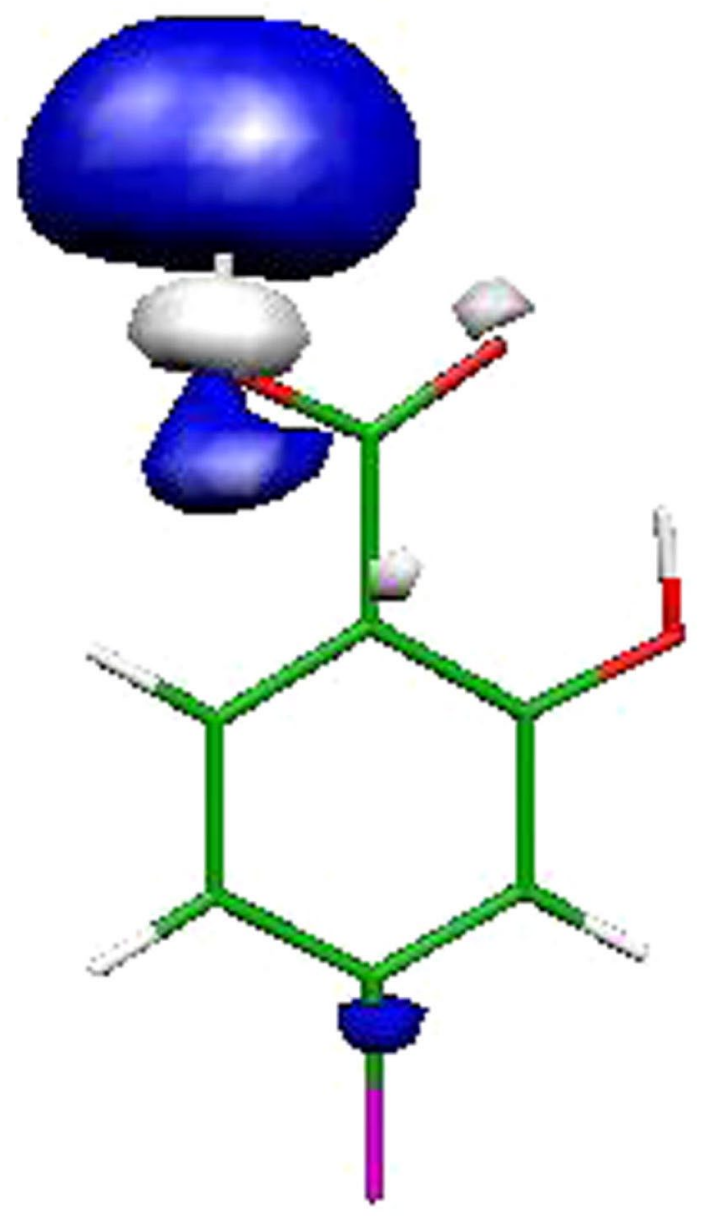

Figure 6. $\sigma^{*}(\mathrm{OH})$ orbital associated with the $-\mathrm{COOH}$ group in salicylic acid.

have substantial spatial overlap with that of the DBS. The most likely candidate is the second lowest empty orbital of $\sigma$ symmetry shown in Figure 6, which lies on the carboxylic acid $\mathrm{OH}$ group and is strongly antibonding between the $\mathrm{O}$ and $\mathrm{H}$ atoms. The excited vibrational levels of a weakly bound DBS lie in the electron scattering continuum, and in our example the valence character of the admixed $\sigma_{2}{ }^{*}(\mathrm{OH})$ orbital permits the levels of the $\mathrm{OH}$ stretch mode to appear as structure in the scattering cross section. Such features are labeled vibrational Feshbach resonances (VFR). ${ }^{20}$ Analogous structures have been observed in the ET spectra of halouracils, ${ }^{21}$ and their role in the DEA cross sections of uracil and thymine have been discussed in detail. ${ }^{22}$ In these cases it is the mixing of the dipole bound state with a $\sigma^{\star}(\mathrm{NH})$ orbital that creates the VFRs. 
The characteristics of the $\sigma_{2}{ }^{*}(\mathrm{OH})$ resonance are expected to be similar to characteristics of the resonance in $\mathrm{HCOOH}$, which has been discussed elsewhere. ${ }^{23}$ The resonance is very broad and not observable by ETS, although it appears in the excitation function of the $\mathrm{OH}$ stretch modes of $\mathrm{HCOOH} .{ }^{24}$ The first $\mathrm{OH}$ stretch level in the chlorosalicylic acids is calculated to lie at $\sim 0.50 \mathrm{eV}$. Consistently, the features labeled VFR lie below this energy, at 0.38 and $0.31 \mathrm{eV}( \pm 0.05)$, with the lower energy associated with the molecule having the higher dipole moment, 3-chlorosalicylic acid, and thus the largest DBS binding energy. No sharp structure appears in the spectrum of 5-chlorosalicylic acid other than the normal curvature leading into the profile of the second $\pi^{*}$ resonance, consistent with its subcritical dipole moment (0.98 D).

Although structure could appear in principle at the $v=2$ or higher vibrational levels of the DBS, such features would be obscured by the $\pi_{2}{ }^{*}$ resonance. Similarly, a VFR at $v=1$ in salicylic acid or benzoic acid is unlikely to be observed because of overlap with the $\pi_{1}{ }^{*}$ resonance.

\section{Phenoxyacetic acid family}

Figure 7 displays the ET spectra of phenoxyacetic acid (a) and several chlorinated congeners including 2,4-D methyl ester (d). In the di- and trichloro compounds, the methyl esters were utilized because of their greater volatility. In contrast to the salicylic acid series, the ring and carboxylic groups have an intervening $-\mathrm{O}-\mathrm{CH}_{2}-$ group that largely decouples their $\pi^{*}$ orbitals from each other. Consequently, the $\pi_{1}^{*}$ and

$\pi_{2}{ }^{*}$ orbitals deriving from the doubly degenerate $\mathrm{e}_{2 \mathrm{u}}$ benzene orbitals undergo relatively small splitting and, analogous to the case in phenol, ${ }^{25} \mathrm{C}_{6} \mathrm{H}_{5}-\mathrm{OH}$, the $\mathrm{b}_{1}$ component is pushed to higher energy, resulting in a LUMO that resembles closely the $\mathrm{a}_{2}$ ring orbital, as verified in our calculations. We anticipate also that the $\pi^{*}$ orbital of the $-\mathrm{COOH}$ group will be relatively constant in energy throughout the series. Table 2 lists the orbitals, the VOEs, and the VAEs.

The orbital labels in Figure 7 reflect our interpretation of the structure in the spectra. In phenoxyacetic acid (a) the close proximity of $\pi_{1}^{*}, \pi_{2}{ }^{*}$, and $\pi^{*}{ }_{\mathrm{COOH}}$ causes only two features to appear below $2 \mathrm{eV}$. It is likely that the overlap of the latter two resonances 


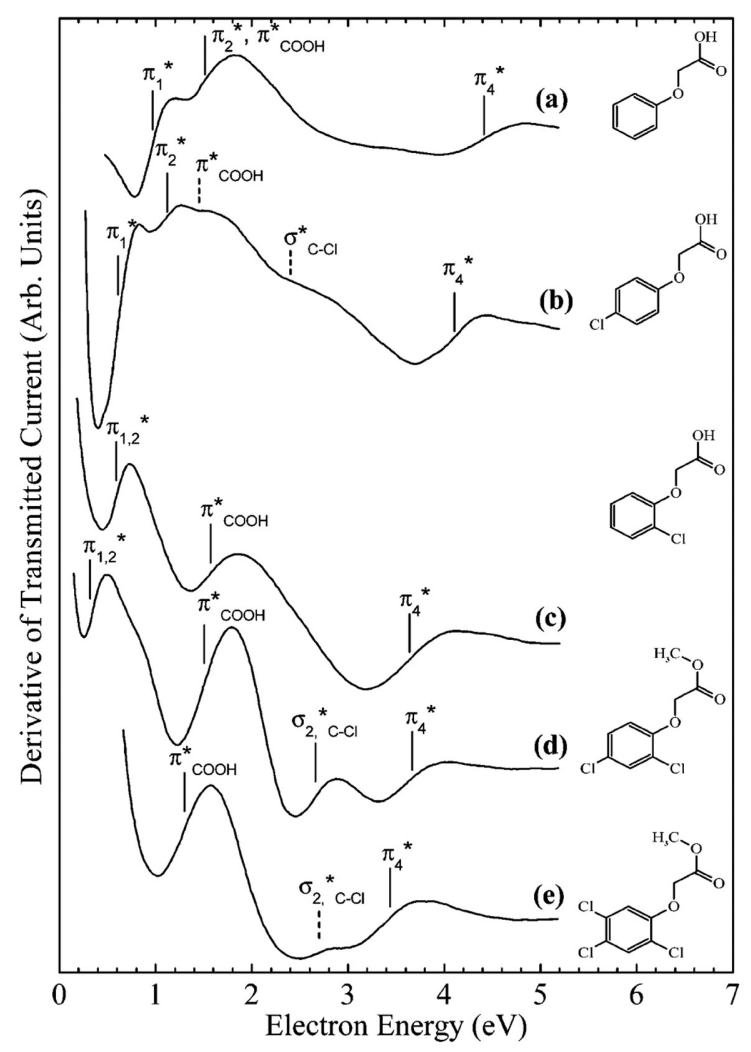

Figure 7. As in Figure 1 for (a) phenoxyacetic acid, (b) (p-chlorophenoxy) acetic acid, (c) (o-chlorophenoxy)acetic acid, (d) (2,4- dichlorophenoxy)acetic acid methyl ester, and (e) (2,3,4- trichlorophenoxy)acetic acid methyl ester.

hides structure arising from $\pi_{2}{ }^{*}$. In support of this, we note that in ( $p$-chlorophenoxy)acetic acid (b), the chlorine substitution has stabilized the ring $\pi^{\star}$ s relative to that of $\pi^{*}{ }_{\mathrm{COOH}}$ revealing evidence for $\pi_{2}{ }^{*}$. No splitting between $\pi_{1}{ }^{*}$ and $\pi_{2}{ }^{*}$ is seen in the $o$-chloro compound (c), although there is some evidence in 2,4-D methyl ester (d) as seen by the change in slope in the derivative signal near 0.8 $\mathrm{eV}$. The latter two spectra appear on sharply declining backgrounds, possibly due to dipole scattering, which would tend to suppress the $\pi_{2}{ }^{*}$ structure. The $\pi_{1,2}{ }^{*}$ resonances could not be observed in the trichloro compound (e). In all cases, the $\pi^{*}{ }_{\text {coor }}$ resonance is prominent, lying between 1.3 and $1.57 \mathrm{eV}$, with the lowest value appearing in the 2,4,5-trichloro compound.

We expect the $\sigma^{\star}(\mathrm{C}-\mathrm{Cl})$ anion states to parallel those found ${ }^{18}$ in chlorobenzene ( $2.42 \mathrm{eV}$ ), 1,3-dichlorobenzene (1.53 and $2.84 \mathrm{eV}$ ), and 1,2,4-trichlorobenzene $(1.17,2.2$, and $3.0 \mathrm{eV})$. However, overlapping 
Table 2. Empty Orbital Designations, VOEs, and VAEs of the Phenoxyacetic Acid Family

\begin{tabular}{|c|c|c|c|}
\hline compound & orbital & $\begin{array}{c}V O E \\
(\mathrm{eV})\end{array}$ & $\begin{array}{l}V A E \\
(\mathrm{eV})\end{array}$ \\
\hline \multirow[t]{4}{*}{ phenoxyacetic acid } & $\pi_{1}^{*}\left[\phi\left(\mathrm{a}_{2}\right)\right]$ & 3.8940 & 0.97 \\
\hline & $\pi_{2}^{*}\left[\phi\left(b_{1}\right)\right]$ & $4 \cdot 3919$ & N.O. ${ }^{a}$ \\
\hline & $\pi_{3}{ }^{*}[\mathrm{COOH}]$ & 5.0123 & 1.51 \\
\hline & $\pi_{4}^{*}[\phi]$ & 10.2696 & \\
\hline \multirow[t]{5}{*}{ ( $p$-chlorophenoxy)acetic acid } & $\pi_{1}^{*}\left[\phi\left(\mathrm{a}_{2}\right)\right]$ & 3.4422 & 0.6 \\
\hline & $\pi_{2}^{*}\left[\phi\left(b_{1}\right)\right]$ & 3.6708 & 1.1 \\
\hline & $\pi_{3}{ }^{*}[\mathrm{COOH}]$ & 4.9144 & $\sim 1.42$ \\
\hline & $\sigma_{1}^{*}[\mathrm{C}-\mathrm{Cl}]$ & $5 \cdot 3960$ & $\sim 2.4$ \\
\hline & $\pi_{4}^{*}[\phi]$ & 9.7825 & \\
\hline \multirow[t]{5}{*}{ (o-chlorophenoxy)acetic acid } & $\pi_{1}^{*}\left[\phi\left(\mathrm{a}_{2}\right)\right]$ & 3.4939 & 0.59 \\
\hline & $\pi_{2}^{*}\left[\phi\left(b_{1}\right)\right]$ & 3.7144 & N. \\
\hline & $\pi_{3}{ }^{*}[\mathrm{COOH}]$ & 4.9824 & 1.57 \\
\hline & $\sigma_{1}^{*}[\mathrm{C}-\mathrm{Cl}]$ & $5 \cdot 3688$ & N.O. \\
\hline & $\pi_{4}^{*}[\phi]$ & 9.8233 & 3.64 \\
\hline \multirow[t]{6}{*}{ (2,4-dichlorophenoxy)acetic acid methyl ester } & $\pi_{1}^{*}\left[\phi\left(\mathrm{a}_{2}\right)\right]$ & 3.1456 & 0.3 \\
\hline & $\pi_{2}^{*}\left[\phi\left(b_{1}\right)\right]$ & 3.3606 & N. \\
\hline & $\sigma_{1}^{*}[\mathrm{C}-\mathrm{Cl}]$ & 4.7049 & N. \\
\hline & $\pi_{3} *[\mathrm{COOH}]$ & 4.9416 & 1.5 \\
\hline & $\sigma_{2} *[\mathrm{C}-\mathrm{Cl}]$ & 6.2341 & 2.66 \\
\hline & $\pi_{4}^{*}[\phi]$ & $9.1131^{b}$ & 3.6 \\
\hline \multirow[t]{7}{*}{$(2,4,5$-trichlorophenoxy)acetic acid methyl ester } & $\pi_{1}^{*}\left[\phi\left(\mathrm{a}_{2}\right)\right]$ & 2.8245 & N. \\
\hline & $\pi_{2}^{*}\left[\phi\left(b_{1}\right)\right]$ & 3.0341 & N.O. \\
\hline & $\sigma_{1}^{*}[\mathrm{C}-\mathrm{Cl}]$ & 4.2205 & N.C \\
\hline & $\pi_{3} *[\mathrm{COOH}]$ & 4.8382 & \\
\hline & $\sigma_{2}^{*}[\mathrm{C}-\mathrm{Cl}]$ & 5.6790 & $\sim 2$ \\
\hline & $\sigma_{3}{ }^{*}[\mathrm{C}-\mathrm{Cl}]$ & 6.5661 & N.O. \\
\hline & $\pi_{4}^{*}[\phi]$ & 8.8301 & 3.4 \\
\hline
\end{tabular}

a. Not observed.

b. Average of 9.1077 and $9.1185 \mathrm{eV}$.

with the $\pi^{*}$ resonances again obscures most of these features, although clear evidence is present in (b), (d), and (e). It is important to realize that these resonances are very broad and the apparent midpoints will be strongly affected by the $\pi^{*}$ resonances on either side. This is particularly true of $\sigma_{2}{ }^{*}$ in 2,4 -D methyl ester which appears 
Table 3. $\sigma^{\star}(\mathrm{C}-\mathrm{Cl})$ VOEs, Scaled VOEs, and VAEs for the Chlorine-Bearing Compounds (All Values in $\mathrm{eV}$ )

\begin{tabular}{lccc} 
compound & $V O E$ & $S V O E^{a}$ & $V A E$ \\
\hline 3-chlorosalicylic acid & 5.3632 & 2.28 & N.O. ${ }^{b}$ \\
4-chlorosalicylic acid & 5.1892 & 2.13 & N.O. \\
5-chlorosalicylic acid & 5.2572 & 2.19 & N.O. \\
(p-chlorophenoxy)acetic acid & 5.3960 & 2.31 & $\sim 2.4$ \\
(o-chlorophenoxy)acetic acid & 5.3688 & 2.29 & N.O. \\
(2,4-dichlorophenoxy)acetic acid methyl ester & 4.7049 & 1.69 & N.O. \\
& 6.2341 & 3.07 & 2.7 \\
(2,4,5-trichlorophenoxy)acetic acid methyl ester & 4.2205 & 1.25 & N.O. \\
& 5.6790 & 2.57 & $\sim 2.7$ \\
& 6.5661 & 3.37 & N.O. \\
2-chloroacetamide & 5.1212 & 2.06 & $\sim 2.3$
\end{tabular}

a. SVOE $=(\operatorname{VOE}-2.83) / 1.11$

$b$. Not observed.

to be narrow. The indicated midpoint, however, is rather close to that found in 1,3-dichlorobenzene. For completeness we show in Table 3 the calculated $\sigma^{*}(\mathrm{C}-\mathrm{Cl})$ VOEs for all the chlorine bearing compounds, their scaled values obtained from ref 7 , and the VAEs, if observed.

While this work was being prepared for publication, Pshenichnyuk and Modelli ${ }^{26}$ reported ETS results in 2,4-D and two other herbicides along with DFT calculations (B3LYP/6-31G(d)) of geometries and VOEs. The latter were scaled using the correlation found in a series of alternating phenyl and acetylenic groups by Scheer and Burrow. ${ }^{27}$ Although the ET spectrum of 2,4-D is close to that observed here in the methyl ester, the lowest observed resonances falling at 0.3 and $0.32 \mathrm{eV}$, respectively, the interpretations differ for their assignments. This arises apparently from the difference in the most stable conformations of the two compounds. The DFT calculations of Pshenichnyuk and Modelli find a planar conformation for neutral 2,4-D, as illustrated in their paper, although a nonplanar conformer is found to be slightly less stable $(0.036 \mathrm{eV})$ at the B3LYP/6-31+G(d) level. ${ }^{26}$ The present HF calculations find a decidedly nonplanar geometry for the methyl ester. It is likely that the bulky methyl group of the latter prevents the planar conformation from having the lowest energy. DFT calculations also confirm the nonplanar geometry of the methyl ester 
[Modelli, private communication]. We note that in 2,4-D, HF and DFT disagree about the most stable conformer, HF preferring the nonplanar geometry.

The geometries described above lead to rather different dispositions of $\pi_{1}^{*}$ and $\pi_{2}{ }^{*}$ in the two molecules. In the planar conformation of 2,4-D, the splitting of these two resonances is larger and the mean energy lower than in the nonplanar 2,4-D methyl ester. This could allow the assignment of the $0.3-0.32 \mathrm{eV}$ feature to be different in these two molecules, that is, to $\pi_{2}{ }^{*}$ in $2,4-\mathrm{D}$ and to $\pi_{1,2}{ }^{*}$ in the methyl ester.

\section{Acetamide family}

Figure 8 displays the ET spectra of acetamide, 2-chloroacetamide, acetanilide, propachlor (2-chloro- $N$-(1-methylethyl)- $N$-phenylacetamide), and metolachlor (2-chloro- $N$-(2-ethyl-6- methylphenyl)- $N$-(2-

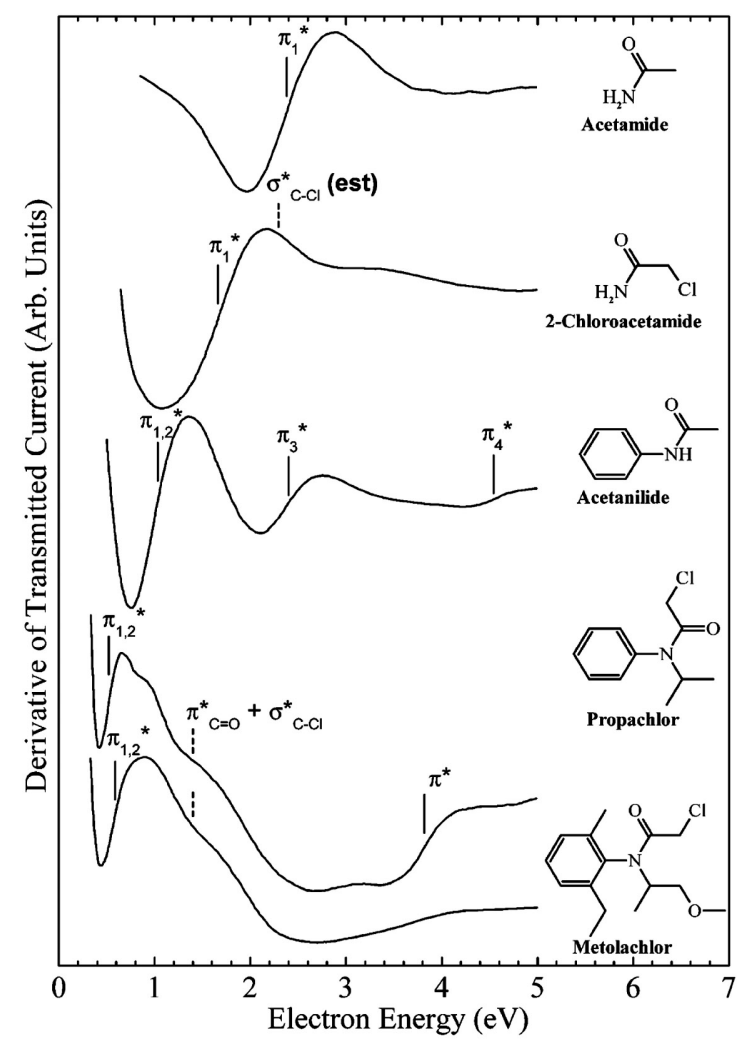

Figure 8. As in Figure 1 for acetamide, 2-chloroacetamide, acetanilide, propachlor, and metolachlor. 
Table 4. Empty Orbital Designations, VOEs, and VAEs of the Acetamide Family

\begin{tabular}{llrr} 
compound & orbital & VOE $(\mathrm{eV})$ & VAE $(\mathrm{eV})$ \\
\hline acetamide & $\pi^{*}[\mathrm{C}=\mathrm{O}]$ & 5.7198 & 2.38 \\
2-chloroacetamide & $\pi^{*}[\mathrm{C}=\mathrm{O}]$ & 5.1729 & 1.66 \\
& $\sigma^{\star}[\mathrm{C}-\mathrm{Cl}]$ & & \\
acetanilide & $\pi_{1}{ }^{*}\left[\phi\left(\mathrm{b}_{1}\right)+\mathrm{C}=\mathrm{O}\right]$ & 5.1212 & $\sim 2.3$ \\
& $\pi_{2}{ }^{*}\left[\phi\left(\mathrm{a}_{2}\right)\right]$ & 3.9103 & 1.03 \\
& $\pi_{3}{ }^{*}\left[\phi\left(\mathrm{b}_{1}\right)-\mathrm{C}=\mathrm{O}\right]$ & 3.9946 & \\
& $\pi_{4}{ }^{*}[\phi]$ & 5.8967 & 2.40 \\
propachlor & $\pi_{1}{ }^{*}\left[\phi\left(\mathrm{b}_{1}\right)\right]$ & 10.362 & 4.54 \\
& $\pi_{2}{ }^{*}\left[\phi\left(\mathrm{a}_{2}\right)\right]$ & 3.3552 & 0.52 \\
& $\pi^{*}[\mathrm{C}=\mathrm{O}]+\sigma^{*}[\mathrm{C}-\mathrm{Cl}]$ & 3.4504 & \\
& $\pi_{4}{ }^{*}[\phi]$ & 4.2042 & $\sim 1.4$ \\
metolachlor & $\pi_{1,2}{ }^{*}[\phi]$ & $9.4995^{a}$ & 3.8 \\
& $\pi^{*}[\mathrm{C}=\mathrm{O}]+\sigma^{*}[\mathrm{C}-\mathrm{Cl}]$ & & 0.59 \\
& & & $\sim 1.4$ \\
\hline
\end{tabular}

a. Average of 9.4451 and $9.5539 \mathrm{eV}$.

methoxy-1-methylethyl)acetamide). The first three of these are planar. In propachlor, the ring is perpendicular to the NCC bonds and the chlorine is not in the $\mathrm{C}=\mathrm{O}$ plane. No calculations were carried out for metolachlor. Table 4 summarizes the results for these compounds in similar fashion as in Table 2.

Acetamide possesses a $\pi^{*}(\mathrm{C}=\mathrm{O})$ resonance at $2.38 \mathrm{eV}$, similar to that of formamide, the smallest compound featuring a peptide-like $\mathrm{NC}=\mathrm{O}$ structure, located at $2.05 \mathrm{eV} .{ }^{28}$ The destabilization in acetamide relative to formamide arises from hyperconjugation of the $\pi^{*}$ orbital with that of the pseudo- $\pi$ of the $-\mathrm{CH}_{3}$ group. The 2-chloroacetamide shows a stabilized $\pi^{*}(\mathrm{C}=\mathrm{O})$ resonance relative to acetamide and a closely overlapping $\sigma^{*}(\mathrm{C}-\mathrm{Cl})$ anion. In previous work in chloroalkanes, 7 it was shown that if the positive peak in the derivative was observable at energy $E_{\mathrm{p}}$, the VAE of the $\sigma^{*}(\mathrm{C}-\mathrm{Cl})$ resonance was approximately given by $E_{\mathrm{p}} / 1.4$. This estimated energy is shown by the dotted line in the ET spectrum.

In the planar acetanilide compound, orbital drawings indicate that the $b_{1}$ component of the benzene LUMO is slightly mixed with that of 
the $-\mathrm{C}=\mathrm{O}$ group. However, the splitting is not enough to separate it from the unmixed $\mathrm{a}_{2}$ component. The $\pi^{*}(\mathrm{C}=\mathrm{O})$ resonance appears almost unshifted from its location in acetamide.

Owing to the nonplanarity of propachlor and metolachlor, the orbital assignments refer only to the local symmetries of the molecular groups. The $\mathrm{b}_{1}$ and $\mathrm{a}_{2}$ components of the benzene LUMO are split only slightly according to the calculations. Because the $\mathrm{C}-\mathrm{Cl}$ bond is not in the $\mathrm{N}-\mathrm{C}-\mathrm{O}$ plane, the local $\pi^{*}$ resonance of the $-\mathrm{C}-\mathrm{O}$ moiety is mixed with the $\sigma^{*}$ resonance associated with the $-\mathrm{C}-\mathrm{Cl}$ group, and the bonding combination of these orbitals leads to a resonance near $1.4 \mathrm{eV}$. Unfortunately, only the positive portion of the derivative signal can be observed, the negative portion being obscured by $\pi_{1,2}{ }^{*}$. The ET spectrum of metolachlor closely resembles that of propachlor and the assignments follow from those given above.

\section{Conclusions}

We have illustrated here the correlations between computed VOEs and their measurable VAE analogs, results which may be of value in studies relating electron reduction rates in herbicides to molecular descriptors, in particular to LUMO properties. As noted before, the correlations are clearly best within a given family, such as that illustrated in Figure 4, which shows a standard deviation of $0.090 \mathrm{eV}$. In Figure 9, we show the correlation for the complete set of observed $\pi^{*}$ anion states for the three families of herbicides and their prototypes. Although the scatter is greater, with a standard deviation of $0.17 \mathrm{eV}$, the regression itself does not differ substantially from that of Figure 4 .

The energies of the $\sigma^{*}(\mathrm{C}-\mathrm{Cl})$ resonances are much more problematic, as they are, for the most part, obscured or partially overlapped by the strong $\pi^{*}$ features. Nevertheless, the scaling derived from studies of the chloroalkanes allows a reasonable estimation of their locations, as shown in Table 3 for the $\sigma^{*}(\mathrm{C}-\mathrm{Cl})$ features that could be identified.

A reviewer notes that given the existence now of many sets of scaling parameters, it is not clear which to use for a new arbitrary compound. For the best accuracy, our choice would be to use the set containing the most structurally similar molecules, and we mention again that a list of scalings known to us may be found in the appendix of 


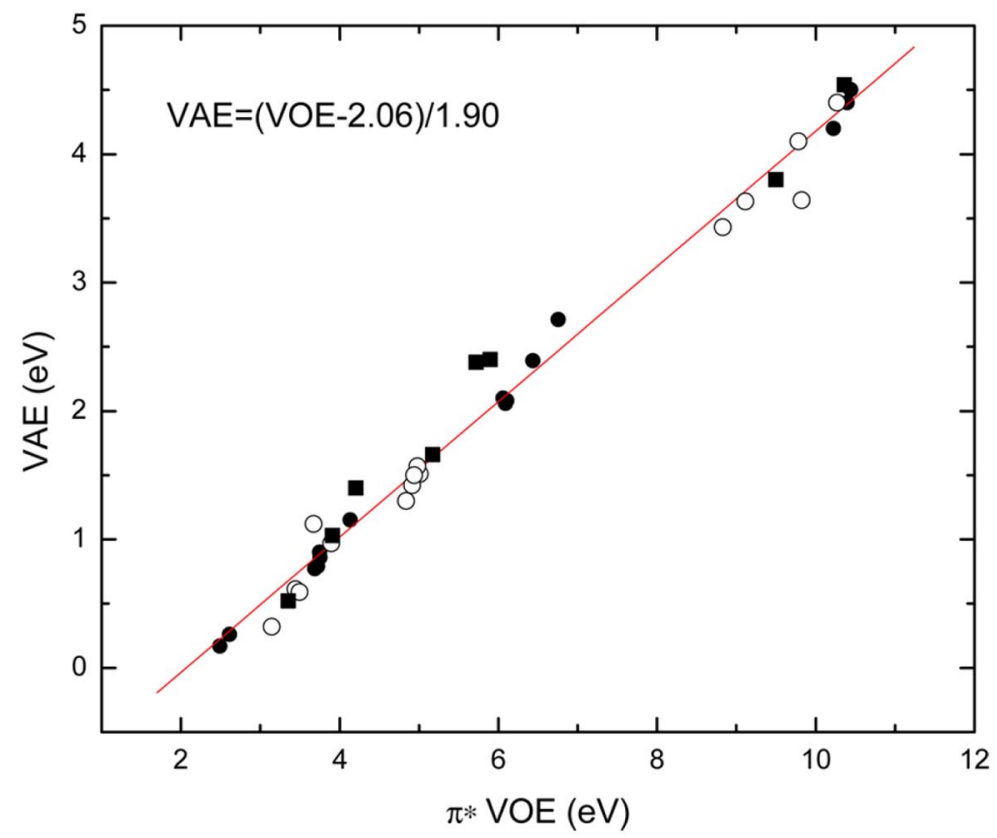

Figure 9. Measured VAEs as a function of $\mathrm{HF} / 6-31 \mathrm{G}(\mathrm{d})$ calculated VOEs of all the compounds. The linear regression and its equation are shown. The salicylic acid family is shown by closed circles, open circles for the phenoxyacetic acid group, and closed squares for the acetamides.

ref 10. On the other hand, a scaling derived from a broad selection of $\pi^{*}$-bearing compounds is given by Modelli ${ }^{29}$ and based on $52 \pi^{*}$ VAEs and calculated (DFT) VOEs. The correlation coefficient $r$ was found to be 0.993. Though not a "universal" scaling, because it does not include $\sigma^{\star}$ orbitals, it will serve as such for $\pi^{\star}$-bearing compounds not included in the existing scalings.

Declaration The authors declare no competing financial interest.

\section{References}

(1) Jordan, K. D.; Burrow, P. D. Acc. Chem. Res. 1978, 11, 341.

(2) Aflatooni, K.; Gallup, G. A.; Burrow, P. D. J. Chem. Phys. 2010, 132, 094306.

(3) Burrow, P. D.; Modelli, A.; Chiu, N. S.; Jordan, K. D. Chem. Phys. Lett. 1981, 82, 270.

(4) Heinrich, N.; Koch, W.; Frenking, G. Chem. Phys. Lett. 1986, 124, 20. 
(5) Chen, D.; Gallup, G. A. J. Chem. Phys. 1990, 93, 8893.

(6) Staley, S. W.; Strnad, J. T. J. Phys. Chem. 1994, 98, 116.

(7) Aflatooni, K.; Gallup, G. A.; Burrow, P. D. J. Phys. Chem. A 2000, 104, 7359.

(8) Pshenichnyuk, S. A.; Asfandiarov, N. L.; Burrow, P. D. Russ. Chem. Bull., Int. Ed. 2007, 56, 1268.

(9) Modelli, A.; Szepes, L. Chem. Phys. 2003, 286, 165.

(10) Burrow, P. D.; Modelli, A. SAR QSAR Environ. Res. 2013, 24, 647.

(11) Burrow, P. D.; Aflatooni, K.; Gallup, G. A. Environ. Sci. Technol. 2000, 34, 3368.

(12) Sanche, L.; Schulz, G. J. Phys. Rev. A 1972, 5, 1672.

(13) Mallory-Smith, C. A.; Retzinger, E. J., Jr. Weed Technol. 2003, 17, 605.

(14) Stamatovic, A.; Schulz, G. J. Rev. Sci. Instrum. 1970, 41, 423.

(15) Johnston, A. R.; Burrow, P. D. J. Electron Spectrosc. Relat. Phenom. 1982, 52, 119.

(16) Burrow, P. D.; Michejda, J. A.; Jordan, K. D. J. Am. Chem. Soc. 1976, 98, 6392.

(17) Schmidt, M. W.; Baldridge, K. K.; Boatz, J. A.; Elbert, S. T.; Gordon, M. S.; Jensen, J. H.; Koseki, S.; Matsunaga, N.; Nguyen, K. A.; Su, S. J.; Windus, T. L.; Dupuis, M.; Montgomery, J. A. J. Comput. Chem. 1993, 14, 1347.

(18) Burrow, P. D.; Modelli, A.; Jordan, K. D. Chem. Phys. Lett. 1986, 132, 441.

(19) Hammer, N. I.; Diri, K.; Jordan, K. D.; Desfrancois, C.; Compton, R. N. J. Chem. Phys. 2003, 119, 3650.

(20) Hotop, H.; Ruf, M. W.; Allan, M.; Fabrikant, I. I. Adv. At., Mol., Opt. Phys. 2003, 49, 85 .

(21) Scheer, A. M.; Aflatooni, K.; Gallup, G. A.; Burrow, P. D. Phys. Rev. Lett. 2004, 92, 068102.

(22) Burrow, P. D.; Gallup, G. A.; Scheer, A. M.; Denifl, S.; Ptasinska, S.; Maerk, T.; Scheier, P. J. Chem. Phys. 2006, 124, 124310.

(23) Gallup, G. A.; Burrow, P. D.; Fabrikant, I. I. Phys. Rev. A 2009, 79, 042701.

(24) Allan, M. J. Phys. At. Mol. Opt. B 2006, 39, 2939.

(25) Jordan, K. D.; Michejda, J. A.; Burrow, P. D. J. Am. Chem. Soc. 1976, 98, 7189.

(26) Pshenichnyuk, S. A.; Modelli, A. Phys. Chem. Chem. Phys. 2013, 15, 9125.

(27) Scheer, A. M.; Burrow, P. D. J. Phys. Chem. B 2006, 110, 17751.

(28) Seydou, M.; Modelli, A.; Lucas, B.; Konate, K.; Desfrancois, C.; Schermann, J. P. Eur. Phys. J. D 2005, 35, 199.

(29) Modelli, A. Phys. Chem. Chem. Phys. 2003, 5, 2923. 